\title{
Acute cytomegalovirus infection in an immunocompetent patient with ulcerative colitis: A case report
}

\author{
TOSHIHIDE MAEDA* , HIROYASU SAKAI*, NORITAKA OZAWA, \\ TOMOHIKO SUGIYAMA, JUN TAKADA, MASAYA KUBOTA, TAKASHI IBUKA, \\ YOHEI SHIRAKAMI, HIROSHI ARAKI and MASAHITO SHIMIZU \\ Department of Gastroenterology, Gifu University Hospital, Gifu 501-1194, Japan
}

Received February 27, 2019; Accepted July 18, 2019

DOI: $10.3892 / \mathrm{etm} .2019 .7823$

\begin{abstract}
Cytomegalovirus (CMV) is a ubiquitous member of the Herpesviridae family that can present with a variety of clinical manifestations, including encephalitis, retinitis, interstitial pneumonia and colitis. These serious symptoms are generally observed as opportunistic infections in immunocompromised hosts, including patients with acquired immunodeficiency syndrome and those receiving steroids and/or immunosuppressants. Symptomatic CMV infections in patients with ulcerative colitis are found in patients treated with steroids and/or immunosuppressants but rarely affect those who are not taking these agents. The present study reported the case of a young patient without concurrent use of immunosuppressive agents for the treatment of ulcerative colitis. The patient presented with acute mononucleosis and colitis caused by primary CMV infection. This was characterized by the presence of atypical lymphocytes and hepatosplenomegaly, elevation of transaminase levels, serology-positive anti-CMV IgM, and CMV antigenemia. Additionally, CMV-positive cells were histologically detected in colonic biopsy specimens. The patient's symptoms and clinical parameters improved following initiation of intravenous ganciclovir. It was concluded that even if patients with ulcerative colitis are not treated with steroids and/or immunosuppressants, significant attention should be paid to acute CMV infections in the context of severe or persistent colonic inflammation.
\end{abstract}

\section{Introduction}

Cytomegalovirus (CMV) is known as a ubiquitous viral agent that affects $40-100 \%$ of adults worldwide (1). Most cases of

Correspondence to: Dr Hiroyasu Sakai, Department of Gastroenterology, Gifu University Hospital, 1-1 Yanagido, Gifu 501-1194, Japan E-mail: sakaih03@gifu-u.ac.jp

${ }^{*}$ Contributed equally

Key words: colitis, cytomegalovirus, mononucleosis, steroids, ulcerative colitis
CMV infections among immunocompetent individuals are asymptomatic and establish a long-term latent infection within the hosts (2). However, serious pathological conditions due to CMV are frequently observed as opportunistic infections in immunocompromised hosts, such as patients with acquired immunodeficiency syndrome (AIDS), malignancies, and those receiving steroids and/or immunosuppressants $(3,4)$.

Recently, a high prevalence of CMV infection of the colon has been reported in patients with inflammatory bowel disease (IBD), including Crohn's disease (CD) and ulcerative colitis (UC) (5-7). Most cases of CMV colitis in patients with IBD can be attributed to the reactivation of a latent CMV infection; active colonic infection induced by a primary CMV infection is rare $(5,8,9)$. In addition, CMV infection of the colon exacerbates IBD, particularly in association with severe or refractory cases under steroid and/or immunosuppressive therapies $(5,6,8,10-12)$. The use of steroids tends to delay the diagnosis of CMV infection by masking abdominal symptoms $(13,14)$. Herein, we present a rare case of acute mononucleosis and colitis caused by a primary CMV infection. Notably, the features of acute CMV infection were observed in an immunocompetent patient with UC without concurrent use of steroids and immunosuppressants.

\section{Case report}

Patient information. A 28-year-old man was diagnosed with active UC (pancolitis type) when he was 20 years old and received remission induction therapy with oral prednisolone (loading dose, $40 \mathrm{mg} /$ day) and 5-aminosalicylic acid (5-ASA) $(4,000 \mathrm{mg} /$ day $)$ for 8 weeks. Since then, he had been on oral 5 -ASA $(4,000 \mathrm{mg} /$ day) treatment for over 7 years. Annual colonoscopy revealed slight inflammation from the rectum to the sigmoid colon; however, no subjective symptoms were noted during the course of the treatment. On September 30, 2016, he developed a headache, rhinorrhea, and myalgia with high-grade fever $\left(38^{\circ} \mathrm{C}\right)$. He was treated with oral acetaminophen $(400 \mathrm{mg}$ twice a day) for 5 days; however, his symptoms did not improve. On October 7, 2016, he presented with bloody diarrhea (5-10 times a day) and lower abdominal discomfort. In addition, computed tomography showed an edematous change in the large intestine that progressed from the rectum to the sigmoid colon. Blood tests also revealed an elevated inflammatory response, 
as indicated by an increase in the number of white blood cells (10,770 cells $/ \mu \mathrm{l})$ and elevated C-reactive protein level $(4.38 \mathrm{mg} /$ dl) and erythrocyte sedimentation rate $(19 \mathrm{~mm} / \mathrm{h})$. A slight elevation of serum aspartate aminotransferase (36 U/l) was observed, but clinically evident liver dysfunction and thrombocytopenia were not. Based on these clinical observations, the patient was suspected to have either infectious colitis or acute exacerbation of UC. Oral levofloxacin (500 mg/day) was administered from October 7 to 11, 2016; however, the patient's symptoms did not improve. He was then admitted to our Hospital on October 11, 2016, for further examination of his condition.

Upon admission, the patient had bloody diarrhea (12 times a day) with a high fever of $38.5^{\circ} \mathrm{C}$ and lower abdominal discomfort. Physical examination revealed slight tenderness at the left lower abdomen and mild hepatosplenomegaly. His chest radiograph and urinalysis results were normal. Blood tests revealed increased numbers of total and atypical lymphocytes (Table I). Mildly elevated aspartate aminotransferase, alanine aminotransferase, and lactate dehydrogenase levels were observed; however, the results of serological tests for hepatitis virus A, $\mathrm{B}$, and $\mathrm{C}$ and Epstein-Barr virus were negative. Colonoscopy showed edematous and erosive changes in the entire mucosa from the rectum to the sigmoid colon (Fig. 1A and B), while colonic mucosa from the cecum to the descending colon was normal. Endoscopic findings from the rectum to the sigmoid colon were consistent with moderately active UC. The bacterial culture results of the patient's blood and stool samples were negative. Clostridium difficile toxins A and B were absent. Notably, the CMV antigenemia assay (CMV pp65 antigen), which is commonly used to detect CMV infection in Japan (4), showed 65 positive cells per 50,000 cells. In addition, the test for anti-CMV IgM antibodies was positive, and the titer of anti-CMV IgG antibodies, which was negative on admission, had increased on the tenth day after admission. Furthermore, hematoxylin and eosin staining of the biopsy specimens from the inflamed colonic mucosa showed cells with inclusion bodies (Fig. 1C). Immunohistochemical examination of these specimens showed CMV positivity (Fig. 1D). Notably, no CMV-positive cells were observed in the biopsy specimens previously collected at the outpatient department. On the basis of these clinical findings, the patient was diagnosed as having acute CMV mononucleosis and colitis on October 14, 2016.

Following the diagnosis of acute CMV mononucleosis and colitis, intravenous ganciclovir (9-[1,3-dihydroxy-2-propoxy] methyl)-guanine (DHPG) (550 mg/day) was administered from October 14 to 19, 2016 (Fig. 2A and B). On the third day, after the initiation of ganciclovir, the hepatic enzymes and atypical lymphocyte levels gradually improved. In addition, the patient's symptoms, including high-grade fever, bloody diarrhea and abdominal discomfort, had subsided. After intravenous ganciclovir was stopped, oral valganciclovir was administered from October 19 to 22, 2016. However, on October 22, 2016, serum CMV pp65 antigen results were found to be negative, and the Seo and Rachmilewitz indices that were used to assess clinical and endoscopic disease activity for $\mathrm{UC}(15,16)$, respectively, had also improved from those seen at admission (Fig. 2C). Therefore, the antiviral treatment for CMV was ended and the patient was discharged on October 22, 2016. Since then, he has been on oral 5-ASA (4,000 mg/day) treatment, and no recurrence of CMV infection has been observed.
Hematoxylin and eosin staining and immunohistochemistry. Histological examination was performed on biopsy specimens fixed with $10 \%$ formalin for $24 \mathrm{~h}$ at room temperature. Paraffin-embedded tissues were cut into $4 \mu \mathrm{m}$-thick sections and deparaffinized. These sections were stained with hematoxylin and eosin or used for immunohistochemistry. For hematoxylin and eosin staining, the sections were stained with $0.1 \%$ hematoxylin solution for $4 \mathrm{~min}$ at room temperature and then stained with $0.1 \%$ eosin Y (cat. no. 058-00062; Wako Pure Chemical Industries, Ltd., Osaka, Japan) solution for $2 \mathrm{~min}$ at room temperature. For immunohistochemistry, the deparaffinized sections were placed in a citrate buffer $(\mathrm{pH}$ 6.0), and then autoclaved at $121^{\circ} \mathrm{C}$ for $1 \mathrm{~min}$ to retrieve the antigen. The sections were then rinsed and blocked with $3 \%$ hydrogen peroxide in methanol for $10 \mathrm{~min}$ to remove endogenous peroxidase activity. Non-specific binding sites were blocked in $0.01 \mathrm{M}$ phosphate-buffered saline (PBS) containing 2\% bovine serum albumin (BSA; cat. no. 019-07494; Wako Pure Chemical Industries, Ltd., Osaka, Japan) for $30 \mathrm{~min}$. Anti-CMV antibody (mouse IgG, cat. no. M0854; Dako, Glostrup, Denmark) diluted to $1: 200$ in $2 \% \mathrm{BSA} / \mathrm{PBS}$ was added to the slides and incubated overnight at $4{ }^{\circ} \mathrm{C}$. Anti-CMV antibody was detected using a biotinylated anti-mouse $\operatorname{IgG}$ (1:300, cat. no. E0433; Dako, Glostrup, Denmark) for $30 \mathrm{~min}$ at room temperature, followed by incubation with avidin-coupled peroxidase (Vectastain ABC kit; Vector Laboratories, Burlingame, CA, USA) for $30 \mathrm{~min}$. The peroxidase binding sites were visualized by staining with 3,3'-diaminobenzidine (DAB) in $50 \mathrm{mM}$ Tris-EDTA buffer and counterstained with hematoxylin.

\section{Discussion}

CMV is a ubiquitous member of the Herpesviridae family that can present with a variety of clinical manifestations, including encephalitis, retinitis, interstitial pneumonia, and colitis (17). These serious symptoms are generally observed as opportunistic infections in immunocompromised hosts, including patients with AIDS and those receiving steroids and/or immunosuppressants (9). Patients with severe or uncontrollable UC often require administration of a steroid or immunosuppressant to decrease disease activity. Therefore, patients with UC are recognized to be at a high risk for symptomatic CMV infections. In the present case, acute mononucleosis and colitis due to a primary CMV infection had been observed in a patient with UC without concurrent immunosuppressive treatments, which is a rare occurrence among patients with UC.

It was previously reported that most cases of CMV colitis observed among patients with IBD can be attributed to the reactivation of a latent CMV infection (8). In this case, CMV-positive cells were observed in biopsy specimens of the colonic mucosa (Fig. 1C and D), indicating the presence of an active colonic infection. Active colonic infection due to CMV is likely to occur by either a primary CMV infection or reactivation of a latent $\mathrm{CMV}$ infection. Another study reported that patients with UC, regardless of their disease activity, have a higher prevalence of anti-CMV IgG than normal control patients (18), indicating a high prevalence of persistent or latent CMV infection among patients with UC. However, in this case, not only were serum anti-CMV IgG and CMV antigenemia present, CMV-positive cells in the biopsy specimens 
Table I. Laboratory data on admission.

\begin{tabular}{|c|c|c|}
\hline Parameter & Data & N.R. \\
\hline WBC (cells/ $\mu \mathrm{l})$ & $11,230^{\mathrm{a}}$ & $3,300-8,600$ \\
\hline Neut $(\%)$ & $22^{\mathrm{b}}$ & $38-74$ \\
\hline Lym (\%) & $50^{\mathrm{a}}$ & $16.5-49.0$ \\
\hline Aty-lymph (\%) & $24^{\mathrm{a}}$ & $0-1$ \\
\hline $\mathrm{RBC}\left(\mathrm{x} 10^{6} / \mu \mathrm{l}\right)$ & 5.29 & $4.35-5.55$ \\
\hline $\mathrm{Hb}(\mathrm{g} / \mathrm{dl})$ & 13.8 & $13.7-16.8$ \\
\hline $\mathrm{Ht}(\%)$ & 41.5 & $40.7-50.1$ \\
\hline Plt $\left(\mathrm{x} 10^{3} / \mu 1\right)$ & 272 & $158-348$ \\
\hline $\mathrm{TP}(\mathrm{g} / \mathrm{dl})$ & 6.7 & $6.6-8.1$ \\
\hline Alb (g/dl) & $3.5^{\mathrm{b}}$ & $4.1-5.1$ \\
\hline AST (U/l) & $87^{\mathrm{a}}$ & $15-30$ \\
\hline ALT (U/l) & $96^{\mathrm{a}}$ & $10-42$ \\
\hline LDH (U/l) & $554^{\mathrm{a}}$ & $124-222$ \\
\hline $\operatorname{ALP}(\mathrm{U} / \mathrm{l})$ & $330^{\mathrm{a}}$ & $106-322$ \\
\hline$\gamma$-GTP $(\mathrm{U} / \mathrm{l})$ & 52 & 13-64 \\
\hline ChE (U/l) & $137^{\mathrm{b}}$ & $201-421$ \\
\hline T.Bil (mg/dl) & 0.4 & $0.4-1.5$ \\
\hline CK (U/l) & $35^{\mathrm{b}}$ & $59-248$ \\
\hline Amy (U/l) & 73 & $44-132$ \\
\hline T-Chol (mg/dl) & $139^{\mathrm{b}}$ & $142-248$ \\
\hline TG (mg/dl) & 108 & $30-117$ \\
\hline UN (mg/dl) & 9.7 & $8.0-20.0$ \\
\hline $\mathrm{Cr}(\mathrm{mg} / \mathrm{dl})$ & 0.83 & $0.65-1.07$ \\
\hline $\mathrm{Na}(\mathrm{mmol} / \mathrm{l})$ & 136 & $134-145$ \\
\hline $\mathrm{K}(\mathrm{mmol} / \mathrm{l})$ & 4.4 & $3.6-4.8$ \\
\hline $\mathrm{Cl}(\mathrm{mmol} / \mathrm{l})$ & 103 & $101-108$ \\
\hline FBS (mg/dl) & 94 & 73-109 \\
\hline HbA1c (\%) & 5.3 & $4.9-6.0$ \\
\hline Cortisol $(\mu \mathrm{g} / \mathrm{dl})$ & 6.8 & $6.2-18.0$ \\
\hline ACTH (pg/ml) & 18 & $7.2-63.3$ \\
\hline CRP (mg/dl) & $7.83^{\mathrm{a}}$ & $<0.14$ \\
\hline \multicolumn{3}{|l|}{ ESR } \\
\hline $30 \min (\mathrm{mm})$ & 4 & N/A \\
\hline $60 \min (\mathrm{mm})$ & $23^{a}$ & $3-15$ \\
\hline CD4 $(\%)$ & 38.4 & $35-65$ \\
\hline CD8 (\%) & 35.4 & $20-38$ \\
\hline CD4/CD8 & 1.09 & $0.4-2.3$ \\
\hline C3 (mg/dl) & 99 & $73-138$ \\
\hline CH50 (U/ml) & 55.5 & $31.6-57.6$ \\
\hline IgG (mg/dl) & 1,587 & $861-1,747$ \\
\hline $\operatorname{IgA}(\mathrm{mg} / \mathrm{dl})$ & 221 & 93-393 \\
\hline $\operatorname{IgM}(\mathrm{mg} / \mathrm{dl})$ & 116 & $33-183$ \\
\hline IgM-HA Ab & $(-)$ & $(-)$ \\
\hline IgM-HBc Ab & $(-)$ & $(-)$ \\
\hline HBs-Ag & $(-)$ & $(-)$ \\
\hline $\mathrm{HCV}$ Ab & $(-)$ & $(-)$ \\
\hline \multicolumn{3}{|l|}{ EBV } \\
\hline VCA-IgM & $<10$ & $<10$ \\
\hline VCA-IgG & $\mathrm{x} 80^{\mathrm{a}}$ & $<10$ \\
\hline EBNA & $\mathrm{x} 40^{\mathrm{a}}$ & $<10$ \\
\hline IgM-CMV Ab & $(+)$ & $(-)$ \\
\hline IgG-CMV Ab & $(-)$ & $(-)$ \\
\hline
\end{tabular}

Table I. Continued.

\begin{tabular}{lcc}
\hline Parameter & Data & N.R. \\
\hline CMV antigenemia & $(+)$ & $(-)$ \\
HIV-1/2 Ab & $(-)$ & $(-)$ \\
HTLV-1 Ab & $(-)$ & $(-)$ \\
T-SPOT.TB & $(-)$ & $(-)$ \\
$\beta$-D-glucan & $<5.0$ & $<5.0$ \\
CD toxin A/B & $(-) /(-)$ & $(-) /(-)$ \\
\hline
\end{tabular}

${ }^{a}$ Increased compared with normal range. ${ }^{b}$ Decreased compared with normal range. ACTH, adrenocorticotrophic hormone; Alb, albumin; ALP, alkaline phosphatase; ALT, alanine aminotransferase; Amy, amylase; AST, aspartate aminotransferase; Aty-lymph, atypical lymphocyte; $\mathrm{CD}$, clostridium difficile; $\mathrm{ChE}$, cholinesterase; $\mathrm{Cl}$, chloride; $\mathrm{CK}$, creatine kinase; $\mathrm{CMV}$, cytomegalovirus; $\mathrm{Cr}$, creatinine; CRP, C-reactive protein; EBNA, Epstein-Barr virus-nuclear antigen; EBV, Epstein-Barr virus; ESR, erythrocyte sedimentation rate; FBS, fasting blood glucose; $\gamma$-GTP, gamma-glutamyl transpeptidase; HA, hepatitis A virus; $\mathrm{Hb}$, hemoglobin; HbA1c, hemoglobin A1c; $\mathrm{HBc}$, hepatitis B virus core; HBs-Ag, hepatitis virus B surface antigen; $\mathrm{HCV}$, hepatitis $\mathrm{C}$ virus; HIV, human immunodeficiency virus; $\mathrm{Ht}$, hematocrit; HTLV-1, human T-cell leukemia virus type 1; IgA, immunoglobulin A; IgG, immunoglobulin G; IgM, immunoglobulin M; K, potassium; LDH, lactate dehydrogenase; Lym, lymphocyte; N/A, not applicable; Na, sodium; Neut, neutrophil; N.R., normal range; Plt, platelet; $\mathrm{RBC}$, red blood cell; T.Bil, total bilirubin; T-Chol, total-cholesterol; TG, triglyceride; T-SPOT.TB, tuberculosis specific interferon $-\gamma$ releasing assay; $\mathrm{TP}$, total protein; $\mathrm{UN}$, urea nitrogen; VCA, viral capsid antigen; WBC, white blood cell.

had been negative before admission. In addition, acute elevation of the anti-CMV IgM titer and positive conversion of anti-CMV IgG were also observed following admission. A primary CMV infection is one that occurs in seronegative subjects without prior specific immunity against CMV (19). Therefore, we concluded that the CMV colitis observed in this patient had resulted from a primary CMV infection and not from the reactivation of a latent CMV infection.

Thus far, primary CMV infections have only rarely been reported in patients with UC without concurrent immunosuppressive treatments. To our knowledge, only 5 case reports have been published since February 1998 (Table II) (20-24), and all cases presented the co-occurrence of CMV colitis. The features of CMV mononucleosis, including fever, atypical lymphocytosis, elevated liver enzymes, and hepatosplenomegaly, were observed in 3 cases $(20,21,23)$. One of the remaining 2 cases showed protein-losing gastroenteropathy (22), whereas the other had toxic megacolon (24). One case had previously undergone short-term steroid treatment for remission induction therapy (21). There were no underlying diseases except UC. Neither was host defense impaired nor were steroids or immunosuppressants administered at the onset of acute CMV infections in all cases. The mechanism of onset of CMV infection has been unknown in most cases (20-22,24), although the immunosuppressive effects of leukocytapheresis (LCAP) have been suggested to be a cause of CMV infection in one study (23). In the present case, prednisolone was previously used for remission induction therapy for UC; however, the patient had not taken any steroids for over 

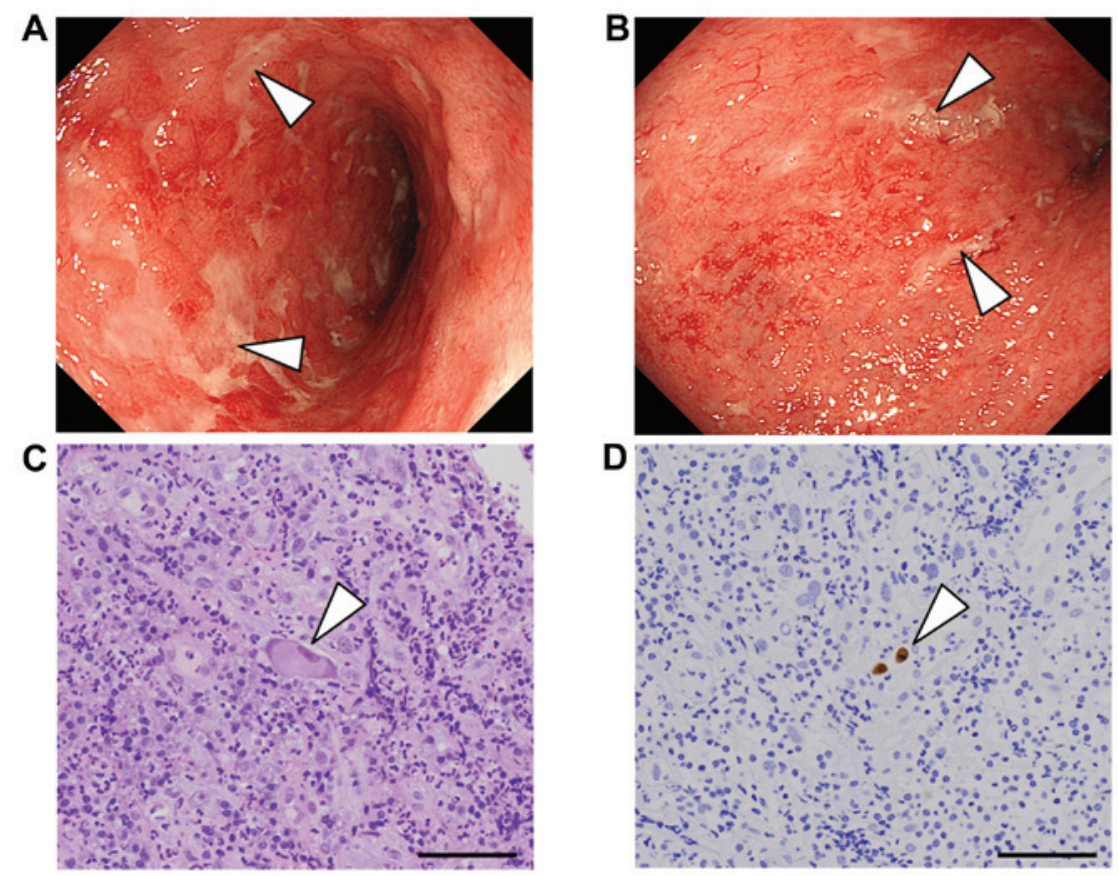

Figure 1. Endoscopic and pathological findings on admission. Endoscopic findings in the (A) rectum and (B) sigmoid colon. Colonoscopy revealed edematous and erosive alterations in the entire mucosa between the rectum and sigmoid colon, as shown by the lack of a visible vascular pattern and mucosal erosion, respectively. The endoscopic findings are consistent with moderately active ulcerative colitis. Arrowheads indicate mucosal erosions. (C) Hematoxylin and eosin staining of the biopsy specimen. The arrowhead indicates a cell with an inclusion body. Scale bar, $50 \mu \mathrm{m}$. (D) Immunostaining for the CMV antigen. The arrowhead indicates CMV antigen-positive cells. Scale bar, $50 \mu \mathrm{m}$. CMV, cytomegalovirus.

A

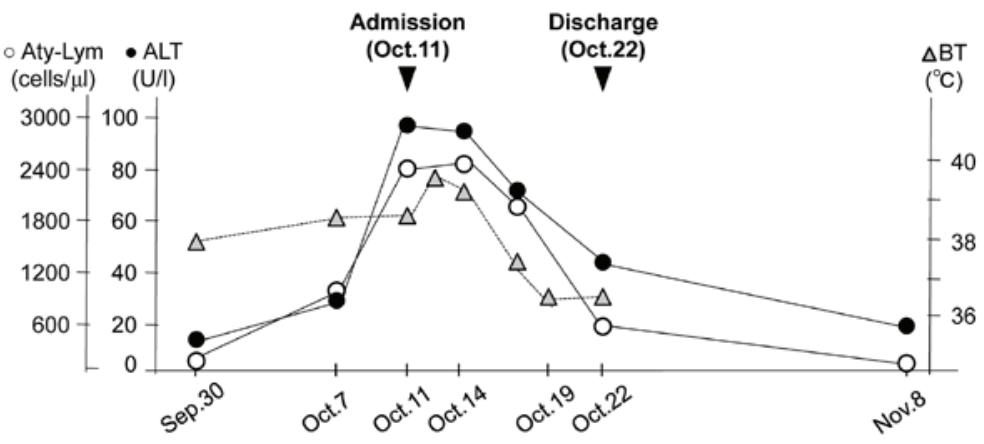

B Levofloxacin $500 \mathrm{mg} /$ day

Ganciclovir $550 \mathrm{mg} /$ day

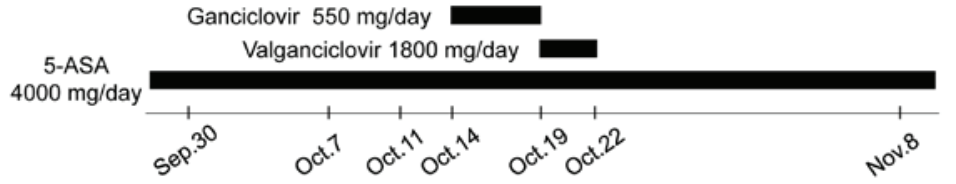

C

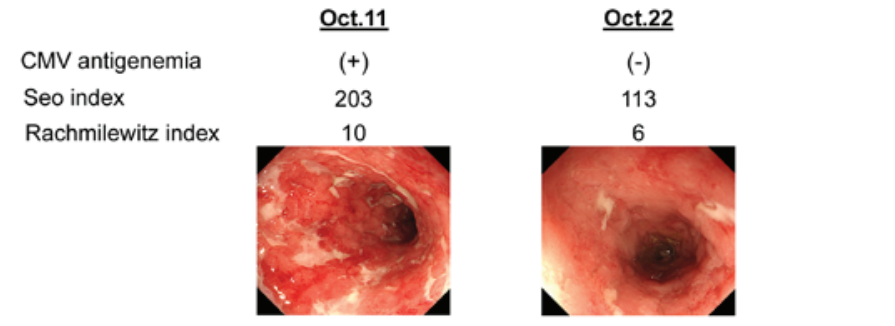

Figure 2. Clinical course of illness of the patient. (A) Clinical course of this patient. (B) Treatment schedule. (C) Comparison of CMV antigenemia and the clinical and endoscopic disease activities at the time of admission and discharge. The Seo index was evaluated by the total score of the patient's symptoms (the presence of bloody stool and bowel movements per day) and laboratory data (ESR, Hb and Alb), indicating the clinical disease activity of UC; total score $<150$, mild; 150-220, moderate; $>220$, severe. A score $<120$ indicates clinical remission (15). The Rachmilewitz endoscopic index is calculated from the total score of colonic mucosal findings [granulation scattering reflected light, visible vascular pattern, vulnerability of mucosa, and mucosal damage (mucus, fibrin, exudate, erosion and ulcer)], indicating the endoscopic disease activity of UC; a total score $<4$ is defined as endoscopic remission (16). Endoscopic findings in the rectum on admission (left) and at the time of discharge (right) are shown. Alb, albumin; ALT, alanine aminotransferase; 5-ASA, 5-aminosalicylic acid; Aty-Lym, atypical lymphocyte; BT, body temperature; CMV, cytomegalovirus; ESR, erythrocyte sedimentation rate; Hb, hemoglobin; Nov., November; Oct., October; Sep., September; UC, ulcerative colitis. 
7 years. Indeed, the serum levels of cortisol and adrenocorticotrophic hormone (ACTH) were within the normal range on admission (Table I), suggesting intact adrenal cortex function. In addition, no abnormal values were observed in both humoral and cellular immunity, as shown by the amount of immunoglobulin and the CD4/CD8 ratio, respectively (Table I). Moreover, comorbidities that may be associated with a degree of immune dysfunction, such as diabetes mellitus or renal failure, were not observed in this patient. Here, immunocompetency was defined as the exclusion of individuals with profound loss of immune function, including patients with AIDS, pharmacologically immunosuppressed patients, and chemotherapy recipients (25). According to this definition and the above-mentioned clinical data, this patient was considered immunocompetent at the onset of acute CMV infection. In addition, the patient had not undergone any procedures such as LCAP. Therefore, consistent with previous reports $(20-22,24)$, the detailed mechanism of the onset of primary CMV infection is also unknown in this case. However, a previous report has shown that a decrease in the seroprevalence of CMV was found among young adults in Japan, suggesting a possible association with increased susceptibility to primary CMV infection in this population (26). Given that this patient's anti-CMV IgG had been negative before admission, this might also, in part, be associated with the onset of primary CMV infection.

Of the 5 cases described in Table II, 3 patients who were treated with the antiviral agent ganciclovir showed marked improvements in all disease manifestations (Table II) $(20,22,23)$. Another case showed recovery with 5-ASA treatment alone (21), and the last case underwent surgical colectomy due to the onset of toxic megacolon (24). It is known that primary CMV infection in immunocompetent individuals is usually asymptomatic or causes a mild mononucleosis-like syndrome, typically resulting in a spontaneous recovery. Therefore, symptomatic treatment is primarily used, and antiviral treatment is not recommended for these populations (27). However, severe tissue-invasive CMV infection with a wide range of manifestations, including colitis, vascular thrombosis, pneumonia, and myocarditis, is occasionally observed, even in immunocompetent adults. Notably, because of the low incidence of such severe CMV infections, usually referred to as CMV disease, its diagnosis and the initiation of targeted therapy tend to be delayed, which leads to high morbidity and mortality due to disease progression. Accordingly, targeted antiviral therapy with ganciclovir or valganciclovir is considered appropriate for the treatment of CMV disease in healthy adults (25). Indeed, 4 patients with UC including the present case have presented with a manifestation of CMV disease, such as colitis, and experienced a dramatic improvement in all disease manifestations after antiviral treatment with ganciclovir (Table II) $(20,22,23)$.

CMV infection in the colon has been reported to exacerbate UC symptoms, resulting in a high incidence of toxic megacolon, colonic perforation, and death $(28,29)$. In fact, a case presented in Table II underwent surgical colectomy due to the onset of toxic megacolon after admission (24). Currently, there is no consensus on how to manage UC patients diagnosed with an active CMV infection. However, considering the role of CMV as an exacerbating factor of UC, the determination of a therapeutic strategy to combat CMV is warranted 
for this patient population. A recent publication reported that both the mortality and surgical rates of patients with UC have improved since the use of ganciclovir and have suggested that ganciclovir is a clearly beneficial therapy for $\mathrm{CMV}$ in patients with UC (30). Indeed, 4 cases of UC, including the present case, have been cured by ganciclovir treatment without any complications (Table II) $(20,22,23)$. However, spontaneous recovery of acute CMV infection has been shown to occur in an immunocompetent patient with UC (21). Moreover, a recent study reported that mild CMV colitis in patients with UC was cured without antiviral therapy (31). Therefore, the use of antiviral agents for acute CMV infection superimposed on immunocompetent UC should be assessed carefully based on the patient's condition, including disease activity and clinical course. Further studies are required to clarify the role of antiviral treatment in immunocompetent patients with UC.

In conclusion, we reported a rare case of acute mononucleosis and colitis caused by primary CMV infection on an immunocompetent patient with UC without concurrent steroids use. Even if patients with UC are not treated with steroids and/or immunosuppressants, clinicians should be aware of the possibility of an acute CMV infection in the context of severe or persistent colonic inflammation.

\section{Acknowledgements}

Not applicable.

\section{Funding}

No funding was received.

\section{Availability of data and materials}

The datasets used and/or analyzed during the present study are available from the corresponding author on reasonable request.

\section{Authors' contributions}

TM and HS collaborated in the conception and design of the study. TM, HS, NO, TS, JT, MK, TI, YS and HA performed the case study, and acquired the data and images of the case. TM, HS and MS performed data analysis and interpretation, and wrote the manuscript. All authors read and approved the final manuscript.

\section{Ethics approval and consent to participate}

Gifu University Hospital, Independent Ethics Committee declares that a single case report does not require an ethical review by the committee. The patient signed an informed consent form for permission to use the clinical data and images.

\section{Patient consent for publication}

Written informed consent was provided by the patient for publication of this study.

\section{Competing interests}

The authors declare that they have no competing interests.

\section{References}

1. de la Hoz RE, Stephens G and Sherlock C: Diagnosis and treatment approaches of CMV infections in adult patients. J Clin Virol 25 (Suppl 2): S1-S12, 2002.

2. Gandhi MK and Khanna R: Human cytomegalovirus: Clinical aspects, immune regulation, and emerging treatments. Lancet Infect Dis 4: 725-738, 2004.

3. Berk T, Gordon SJ, Choi HY and Cooper HS: Cytomegalovirus infection of the colon: A possible role in exacerbations of inflammatory bowel disease. Am J Gastroenterol 80: 355-360, 1985.

4. Ikeda K, Nakajima S, Tanji K, Hirai T, Uomori K, Morimoto S, Tomita S, Fukunaga M, Tamura N and Sekigawa I: Intestinal perforation due to hemorrhagic Cytomegalovirus enteritis in a patient with severe uncontrolled lupus nephritis: A case and review of the literature. Rheumatol Int 37: 1395-1399, 2017.

5. Babyatsky MW, Keroack MD, Blake MA, Rosenberg ES and Mino-Kenudson M: Case records of the Massachusetts General Hospital. Case 35-2007. A 30-year-old man with inflammatory bowel disease and recent onset of fever and bloody diarrhea. N Engl J Med 357: 2068-2076, 2007.

6. Dimitroulia E, Spanakis N, Konstantinidou AE, Legakis NJ and Tsakris A: Frequent detection of cytomegalovirus in the intestine of patients with inflammatory bowel disease. Inflamm Bowel Dis 12: 879-884, 2006.

7. Galiatsatos P, Shrier I, Lamoureux E and Szilagyi A: Meta-analysis of outcome of cytomegalovirus colitis in immunocompetent hosts. Dig Dis Sci 50: 609-616, 2005.

8. Papadakis KA, Tung JK, Binder SW, Kam LY, Abreu MT, Targan SR and Vasiliauskas EA: Outcome of cytomegalovirus infections in patients with inflammatory bowel disease. Am J Gastroenterol 96: 2137-2142, 2001.

9. Khan TV and Toms C: Cytomegalovirus colitis and subsequent new diagnosis of inflammatory bowel disease in an immunocompetent host: A case study and literature review. Am J Case Rep 17: 538-543, 2016.

10. Kishore J, Ghoshal U, Ghoshal UC, Krishnani N, Kumar S, Singh M and Ayyagari A: Infection with cytomegalovirus in patients with inflammatory bowel disease: Prevalence, clinical significance and outcome. J Med Microbiol 53: 1155-1160, 2004.

11. Pillet S, Pozzetto B and Roblin X: Cytomegalovirus and ulcerative colitis: Place of antiviral therapy. World J Gastroenterol 22: 2030-2045, 2016.

12. Römkens TE,Bulte GJ,Nissen LH and Drenth JP: Cytomegalovirus in inflammatory bowel disease: A systematic review. World $\mathrm{J}$ Gastroenterol 22: 1321-1330, 2016.

13. Ohnuki Y, Moriya Y, Yutani S, Mizuma A, Nakayama T, Ohnuki Y, Uda S, Inomoto C, Yamamoto S, Nakamura N and Takizawa S: Eosinophilic granulomatosis with polyangiitis (churg-strauss syndrome) complicated by perforation of the small intestine and cholecystitis. Intern Med 57: 737-740, 2018.

14. Ikeda K, Takasaki Y and Sekigawa I: Rapid onset of small intestinal perforation after successful steroid treatment in eosinophilic granulomatosis with polyangiitis. Mod Pheumatol 26: 968-970, 2016.

15. Seo M, Okada M, Yao T, Ueki M, Arima S and Okumura M: An index of disease activity in patients with ulcerative colitis. Am J Gastroenterol 87: 971-976, 1992.

16. Rachmilewitz D: Coated mesalazine (5-aminosalicylic acid) versus sulphasalazine in the treatment of active ulcerative colitis: A randomized trial. BMJ 298: 82-86, 1989.

17. Eddleston M, Peacock S, Juniper M and Warrell DA: Severe cytomegalovirus infection in immunocompetent patients. Clin Infect Dis 24: 52-56, 1997.

18. Farmer GW, Vincent MM, Fuccillo DA, Horta-Barbosa L, Ritman S, Sever JL and Gitnick GL: Viral investigations in ulcerative colitis and regional enteritis. Gastroenterology 65: 8-18, 1973.

19. Ho M: The history of cytomegalovirus and its disease. Med Microbiol Immunol 197: 65-73, 2008.

20. Moonka D, Furth EE, MacDermott RP and Lichtenstein GR: Pouchitis associated with primary cytomegalovirus infection. Am J Gastroenterology 93: 264-266, 1998.

21. Rachima C, Maoz E, Apter S, Thaler M, Grossman E and Rosenthal T: Cytomegalovirus infection associated with ulcerative colitis in immunocompetent individuals. Postgrad Med J 74: 486-489, 1998.

22. Kraus M, Meyenberger C and Suter W: Generalized intestinal CMV infection with protein-losing syndrome in ulcerative colitis. Schweiz Med Wochenschr 130: 1600-1605, 2000. 
23. Osaki R, Andoh A, Tsujikawa T, Ogawa A, Koizumi Y, Nakahara T, Hata K, Sasaki M, Saito Y and Fujiyama Y: Acute cytomegalovirus infection superimposed on corticosteroid-naïve ulcerative colitis. Intern Med 47: 1341-1344, 2008.

24. Inoue $\mathrm{K}$, Wakabayashi N, Fukumoto K, Yamada S, Bito N Yoshida N, Katada K, Uchiyama K, Ishikawa T,Handa O, et al: Toxic megacolon associated with cytomegalovirus infection in a patient with steroid-naïve ulcerative colitis. Intern Med 51: 2739-2743, 2012.

25. Lancini D, Faddy HM, Flower R and Hogan C: Cytomegalovirus disease in immunocompetent adults. Med J Aust 201: 578-580, 2014.

26. Takeda N, Isonuma $H$, Sekiya $S$, Ebe $T$, Matsumoto $T$ and Watanabe K: Studies of anti-cytomegalovirus IgG antibody rate and cytomegalovirus mononucleosis in adults. Kansenshogaku Zasshi 75: 775-779, 2001

27. Pokorska-Śpiewak M, Niezgoda A, Gołkowska M, Czech-Kowalska J, Gruszfeld D, Dobrzańska A, Styczyński J and Marczyńska M: Recommendations for the diagnosis and treatment of CMV infections. Polish Society of epidemiology and infectious disease. Przegl Epidemiol 70: 297-310, 2016.
28. Cooper HS, Raffensperger EC, Jonas L and Fitts WT Jr: Cytomegalovirus inclusions in patients with ulcerative colitis and toxic dilation requiring colonic resection. Gastroenterology 72 : 1253-1256, 1977

29. Sidi S, Graham JH, Razvi SA and Banks PA: Cytomegalovirus infection of the colon associated with ulcerative colitis. Arch Surg 114: 857-859, 1979.

30. Pfau P, Kochman ML, Furth EE and Lichtenstein GR Cytomegalovirus colitis complicating ulcerative colitis in the steroid-naïve patient. Am J Gastroenterol 96: 895-899, 2001.

31. Kopylov U, Sasson G, Geyshis B, Oikawa MT, Barshack I, Eliakim R and Ben-Horin S: Cytomegalovirus positive ulcerative colitis: A single center experience and literature review. World J Gastrointest Pathophysiol 4: 18-23, 2013. 